\title{
Cardiac injury after acute carbon monoxide poisoning and its clinical treatment scheme
}

\author{
SHOUMENG GENG ${ }^{1 *}$, XIAOYAN HAO ${ }^{2 *}$, HAICANG XU ${ }^{3}$, JIAN YAO $^{1}$, \\ DONGYONG $\mathrm{HE}^{1}$, HUI XIN ${ }^{4}$, XINGJI GONG ${ }^{1}$ and RUI ZHANG ${ }^{4}$ \\ ${ }^{1}$ Department of Emergency, The Affiliated Hospital of Qingdao University, Qingdao, Shandong 266000; \\ ${ }^{2}$ Department of Emergency, Qingdao Municipal Hospital, Qingdao, Shandong 266011; Departments of ${ }^{3}$ Pathology \\ and ${ }^{4}$ Cardiology, The Affiliated Hospital of Qingdao University, Qingdao, Shandong 266000, P.R. China
}

Received November 19, 2019; Accepted December 11, 2019

DOI: $10.3892 / \mathrm{etm} .2020 .8801$

\begin{abstract}
This study was designed to investigate cardiac injury after acute carbon monoxide poisoning and its clinical treatment scheme. Seventy patients with moderate and severe acute carbon monoxide poisoning (ACOP) admitted from January 2017 to December 2018 into The Affiliated Hospital of Qingdao University were regarded as a research group (RG), and another 30 healthy adults undergoing physical examination in the hospital during the same period were selected as a control group (CG). Thirty-five patients in the $R G$ who received hyperbaric oxygen therapy were considered as group $\mathrm{A}$, and 35 patients who received extracorporeal membrane oxygenation therapy were considered as group B. The effective rates and complications of the two groups after treatment were compared. The concentrations of creatine kinase isoenzyme (CK-MB) and lactate dehydrogenase $(\mathrm{LDH})$ of myocardial enzymes at different time points before and after treatment were detected. Expression of miR-30a in the blood of experimental subjects was detected by time-fluorescence quantitative PCR, and the relationship between miR-30a expression and ACOP patients was analyzed. Patients in groups A and B achieved obvious efficacy, but the effective rate and incidence rate of complications in the extracorporeal membrane oxygenation (ECMO) group were better than those in the hyperbaric oxygen group. The concentrations of CK-MB and LDH in group A and group B were significantly higher than those in control group
\end{abstract}

Correspondence to: Dr Rui Zhang, Department of Cardiology, The Affiliated Hospital of Qingdao University, 1677 Wutaishan Road, Qingdao Development Zone, Qingdao, Shandong 266000, P.R. China

E-mail: rbi4lm@163.com

${ }^{*}$ Contributed equally

Key words: cardiac injury, acute carbon monoxide poisoning, hyperbaric oxygen, ECMO miR-30a
$(\mathrm{P}<0.01)$. The expression level of miR-30a in the RG was significantly higher than that in the control group $(\mathrm{P}<0.05)$. Both hyperbaric oxygen therapy and ECMO therapy have obvious efficacy on ACOP patients, but the latter is better than the former. The expression level of miR-30a in blood of ACOP patients increased significantly, which is positively correlated with myocardial injury, and it decreased after treatment. It is believed that miR-30a can provide a reference index for early diagnosis and prediction of disease progression and prognosis in cardiac injury of ACOP.

\section{Introduction}

As a colorless and tasteless gas, carbon monoxide $(\mathrm{CO})$ is difficult to find when it overflows. The concentration of $\mathrm{CO}$ exceeding $35 \mathrm{ppm}$ will endanger human health (1). Acute carbon monoxide poisoning (ACOP) is a common acute disease in clinical practice. Its clinical manifestations range from headache, dizziness to coma and even death with a mortality rate of 1-3\%. A considerable number of patients have longterm neurological and emotional sequelae after treatment (2). Its pathogenesis is not completely clear and its physiological mechanism is mainly hypoxia stress. In ACOP, the main harm is to organs with high oxygen demand, including heart and brain, and the severity of the disease may be related to different concentrations of $\mathrm{CO}$ exposure and duration (3). Among them, myocardial injuries such as angina pectoris and myocardial infarction (4) are common in ACOP patients and are related to the increase of long-term mortality rate (5). At present, clinical treatment for ACOP includes hyperbaric oxygen therapy on the basis of symptomatic treatment, which can effectively reduce the mortality rate of patients (6). However, due to the lack of efficacy basis, the application of hyperbaric oxygen to ACOP patients still lacks relevant evidence (7). In recent years, due to the development of extracorporeal circulation technology, extracorporeal membrane oxygenation (ECMO) technology has been gradually applied to the treatment of ACOP, and Simonsen et al (8) reported that ECMO treatment can improve the survival rate of ACOP patients with cardiogenic shock, but its exact efficacy is currently less studied.

Physiological autophagy has a protective effect in myocardial ischemia, and miR-30a can maintain autophagy reaction 
of myocardial cells after hypoxia (9), so miR-30a is bound to have an important connection with cardiac injury of ACOP. Therefore, this study judged myocardial injury by detecting the miR-30a expression level before and after ACOP patients received hyperbaric oxygen therapy and ECMO respectively, and detected myocardial enzymes: creatine kinase isoenzyme (CK-MB) and lactate dehydrogenase (LDH). The aim was further confirm the efficacy and mechanism of the two treatment methods, to judge which treatment method is more suitable for clinical use, and to provide more reliable reference opinions for the future treatment of such diseases.

\section{Patients and methods}

Clinical data. Seventy patients with moderate and severe ACOP admitted to The Affiliated Hospital of Qingdao University (Qingdao, China) from January 2017 to December 2018 were studied. On the basis of routine treatment, they were divided into group A (treated with hyperbaric oxygen) with 35 cases and group B (treated with ECMO) with 35 cases according to different combined treatment methods. Another 30 healthy adults who underwent physical examination in the hospital were selected as the control group (CG). The subjects were aged 25-60 years, with an average age of 41.53 \pm 8.71 years. This study was approved by the Ethics Committee of The Affiliated Hospital of Qingdao University, and all patients and their families signed an informed consent.

Inclusion and exclusion criteria. All the patients involved in this experiment had a history of $\mathrm{CO}$ exposure and met the diagnostic criteria of moderate or severe ACOP. Exclusion criteria were as follows: i) Mild ACOP patients; ii) patients with a history of drug allergy; iii) patients with mental disorders; iv) patients with severe cardiac, cerebral and renal insufficiency before suffering from this disease; v) those who were restless and could not cooperate with the treatment; vi) patients with incomplete pathological data; vii) patients with coagulation dysfunction.

Methods. On the basis of routine treatment, all patients in group A received hyperbaric oxygen therapy: stable pressurization for $20 \mathrm{~min}$, stable oxygen inhalation for $60 \mathrm{~min}$, intermittent rest for $5 \mathrm{~min}$, gradual decompression for $20 \mathrm{~min}$, hyperbaric oxygen chamber treatment pressure of 2-2.5 ATA, and hyperbaric oxygen therapy once a day. Patients in group B received ECMO treatment: The vein-artery (V-A) auxiliary mode was selected, and ECMO was composed of a temperature-variable water tank, a centrifugal pump, an oxygenator and heparin-coated cannulas. They all underwent ipsilateral femoral artery and femoral vein incision, heparin-coated cannulas of 15-17FR and 19-21FR were inserted, appropriate flow paths were set, blood supply to distal limbs of the same side was ensured, and ECMO was managed and removed according to the guidelines.

Altogether $3 \mathrm{ml}$ of peripheral blood in all subjects was taken at different time points before and after treatment, while from those in the CG $2 \mathrm{ml}$ of venous blood was taken during physical examination. Myocardial enzyme detection: serum was taken by low-speed centrifugation at $4^{\circ} \mathrm{C}$ for $20 \mathrm{~min}$ at $1,000 \mathrm{x} \mathrm{g}, \mathrm{CK}-\mathrm{MB}$ and $\mathrm{LDH}$ levels were detected by Dx800 automatic biochemical immune analyzer (Beckman Coulter, Inc.) and enzyme-linked immunosorbent assay (ELISA). CK-MB and LDH kits were from Shanghai Yubo Biotechnology Co., Ltd. miR-30a detection: Real-time fluorescence quantitative PCR (RT-PCR) method was used to detect the expression of target miR-30a (kit from Xiamen Huijia Biotechnology Co., Ltd., XWCPK3043). Trizol kit (Shenyang Wanlei Biotechnology Co., Ltd.) was used to extract the total RNA of cells, and specific steps were strictly carried out in accordance with the instructions. The extracted RNA was reverse transcribed to obtain cDNA, which was used as a template for experiments. The reaction conditions were as follows: pre-denaturation at $95^{\circ} \mathrm{C}$ for $30 \mathrm{sec}$, denaturation at $95^{\circ} \mathrm{C}$ for $5 \mathrm{sec}$, and denaturation at $60^{\circ} \mathrm{C}$ for $20 \mathrm{sec}$. Melting conditions: $95^{\circ} \mathrm{C}, 0 \mathrm{sec} ; 65^{\circ} \mathrm{C}, 15 \mathrm{sec}$; and $95^{\circ} \mathrm{C}$, 0 sec. Samples in each group were repeated 3 times, internal reference was performed using U6, and data analysis was performed using $2^{-\Delta \mathrm{Ct}}$ method. The primer sequence was designed and synthesized by China Thermo Fisher Scientific, Inc., as shown in Table I.

Observation index and efficacy judgment standard. The efficacy evaluation was divided into three grade standards, namely markedly effective, effective and ineffective.

Markedly effective: The symptoms and signs of the patients were obviously improved, their consciousness was clear, and the electroencephalogram basically returned to normal. Effective: The symptoms and signs of the patients were improved and the consciousness recovered basically, but the EEG examination was still abnormal. Ineffective: The symptoms and signs of the patients were not improved, even aggravated or presenting serious complications.

The effective rate and complications of patients in the two groups two weeks after treatment were compared. The CK-MB and LDH levels as well as miR-30a expression in blood samples of subjects in the three groups at different time points before and after treatment were observed.

Statistical methods. SPSS24.0 statistical software was used to analyze and process the data, GraphPad 5 software package was used to draw relevant images, the counting data were expressed in the form of rate, and Chi-square test was used for comparison between groups. The measurement data were expressed as mean \pm standard deviation. Independentsamples t-test was used for inter-group comparison, one-way analysis of variance was used for multi-group comparison, LSD t-test was used for post hoc pairwise comparison, repeated measures analysis of variance was used for multitime point expression, and Bonferroni test was used for back testing. The diagnostic value was analyzed by ROC curve. $\mathrm{P}<0.05$ was considered to indicate a statistically significant difference.

\section{Results}

Comparison of clinical data. There was no significant difference in age, sex, white blood cell count, exposure time, typing, smoking, and family history of heart disease between the RG, $\mathrm{CG}$ and groups $\mathrm{A}$ and $\mathrm{B}(\mathrm{P}>0.05)$, proving that subjects in the three groups were comparable (Table II). 
Table I. miR-30a primer sequences.

\begin{tabular}{llc}
\hline Sequence & \multicolumn{1}{c}{ miR-30a } & U6 \\
\hline Forward & 5'-ACGGGCGCAACTGTAAACATCC-3' & 5'-GACATCAAGAAGGTGGTGAAGC-3' \\
Reverse & 5'-CAGTGCAGGGTCCGAGGTAT-3' & 5'-TGTCATTGAGAGCAATGCCAGC-3' \\
\hline
\end{tabular}

Table II. Comparison of clinical data $[\mathrm{n}(\%)]$.

\begin{tabular}{|c|c|c|c|c|c|}
\hline Parameters & Group A $(n=35)$ & Group B $(n=35)$ & Control group $(n=30)$ & $\chi^{2}$ or $\mathrm{F}$ value & $\mathrm{P}$-value \\
\hline Age & $39.88 \pm 6.41$ & $42.25 \pm 7.56$ & $43.18 \pm 7.29$ & 1.910 & 0.154 \\
\hline Sex & & & & 2.190 & 0.335 \\
\hline Male & $14(40.00)$ & $19(54.29)$ & 17 (56.67) & & \\
\hline Female & $21(60.00)$ & $16(45.71)$ & $13(43.33)$ & & \\
\hline White blood cell count $\left(10^{9} / 1\right)$ & $8.53 \pm 2.64$ & $8.26 \pm 2.12$ & $7.51 \pm 1.96$ & 1.715 & 0.185 \\
\hline Exposure time (h) & $6.43 \pm 4.57$ & $6.67 \pm 5.19$ & & 0.205 & 0.838 \\
\hline Typing & & & & 0.245 & 0.621 \\
\hline Moderate & $23(65.71)$ & $21(60.00)$ & & & \\
\hline Severe & $12(34.29)$ & $14(40.00)$ & & & \\
\hline Family history of heart disease & & & & 0.688 & 0.709 \\
\hline Yes & $5(14.29)$ & $6(17.14)$ & $3(10.00)$ & & \\
\hline No & $30(85.71)$ & $29(82.86)$ & $27(90.00)$ & & \\
\hline Smoking & & & & 1.476 & 0.478 \\
\hline Yes & $11(31.43)$ & $15(42.86)$ & $9(30.00)$ & & \\
\hline No & $24(68.57)$ & $20(57.14)$ & $21(70.00)$ & & \\
\hline
\end{tabular}

Comparison of the effective rates of patients in the two groups. The total effective rate of patients in group A was $71.43 \%$, and that of patients in group B was $91.43 \%$. Compared with patients in the two groups, those in group B were significantly higher than those in group $\mathrm{A}(\mathrm{P}<0.05)$ (Table III).

Comparison of the incidence rate of complications between the two groups. The two groups were compared for delayed encephalopathy, myocardial injury, urinary tract infection, skin dystrophy and other complications. Analysis revealed that the incidence rate of complications in group B $(14.29 \%)$ was significantly lower than that in group A $(37.14 \%)(\mathrm{P}<0.05)$ (Table IV).

Comparison of $C K-M B$ and LDH levels at different time points before and after treatment. There was no significant difference in CK-MB and LDH concentrations between group A, group $\mathrm{B}$ and control group before treatment $(\mathrm{P}>0.05)$. Three days, seven days and two weeks after treatment, the levels of CK-MB and LDH in group A were significantly lower than those in group $\mathrm{B}(\mathrm{P}<0.05)$ (Fig. 1).

Detection of miR-30a expression level. Blood samples of 100 subjects were collected before, and seven days after treatment to detect the expression level of miR-30a, and the expression level of miR-30a in ACOP patients before treatment was significantly higher than that in normal samples
Table III. Comparison of therapeutic efficiency.

\begin{tabular}{lcccc}
\hline Efficiency & $\begin{array}{c}\text { Group A } \\
(\mathrm{n}=35)\end{array}$ & $\begin{array}{c}\text { Group B } \\
(\mathrm{n}=35)\end{array}$ & $\chi^{2}$ value & P-value \\
\hline Markedly effective & $14(40.00)$ & $18(51.43)$ & & \\
Effective & $11(31.43)$ & $14(40.00)$ & & \\
Ineffective & $10(28.57)$ & $3(8.57)$ & & \\
Total effective rate & $71.43 \%$ & $91.43 \%$ & 4.629 & 0.031 \\
\hline
\end{tabular}

$(\mathrm{P}<0.01)$, at seven days after treatment it was significantly lower than that before treatment, and the reduction level in group $\mathrm{B}$ was better than that in group $\mathrm{A}(\mathrm{P}<0.05)$ (Fig. 2).

Diagnostic value of miR-30a in myocardial injury of ACOP. ROC curve analysis showed that the area under the curve (AUC) was 0.870 . When the maximum cut-off value was 3.807, the sensitivity and specificity of miR-30a in diagnosing myocardial injury of ACOP were 68.57 and $96.67 \%(\mathrm{P}<0.01)$ (Fig. 3).

Correlation analysis between the miR-30a expression level and clinicopathology of ACOP patients. The expression level of miR-30a was not significantly correlated with age, sex, 
Table IV. Incidence rate of complications.

\begin{tabular}{lccc}
\hline Factors & Group A $(\mathrm{n}=35)$ & Group B $(\mathrm{n}=35)$ & $\chi^{2}$ value \\
\hline Delayed encephalopathy & $5(14.29)$ & $3(8.57)$ & \\
Myocardial injury & $4(11.43)$ & $2(5.71)$ & \\
Urinary tract infection & $2(5.71)$ & $0(0.00)$ & \\
Cutaneous dystrophy & $2(5.71)$ & $0(0.00)$ & 4.786 \\
Incidence rate of complications & $37.14 \%$ & $14.29 \%$ & 0.029
\end{tabular}

A

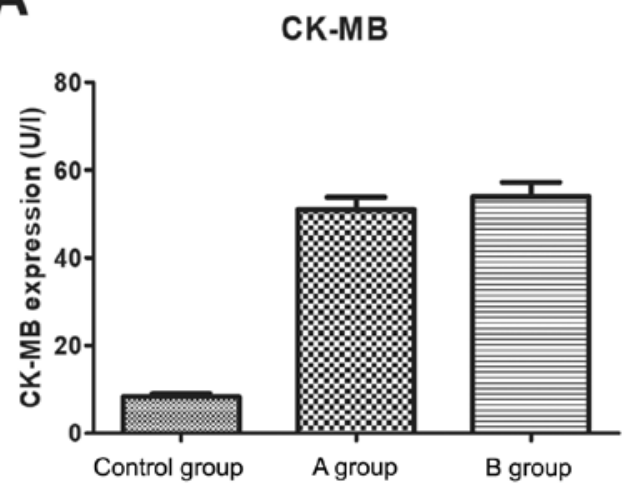

C

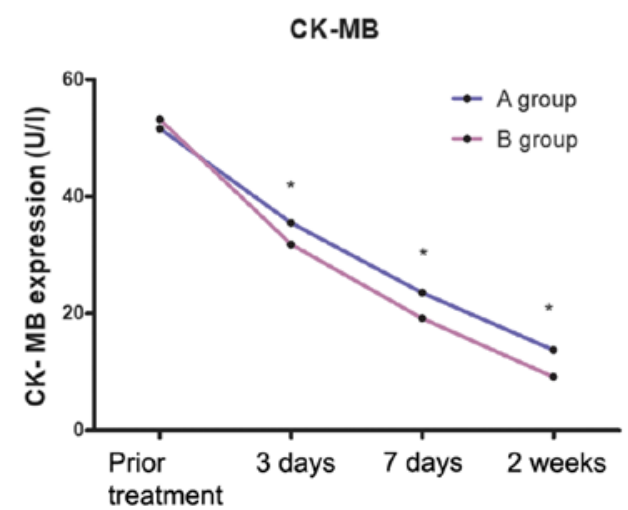

B

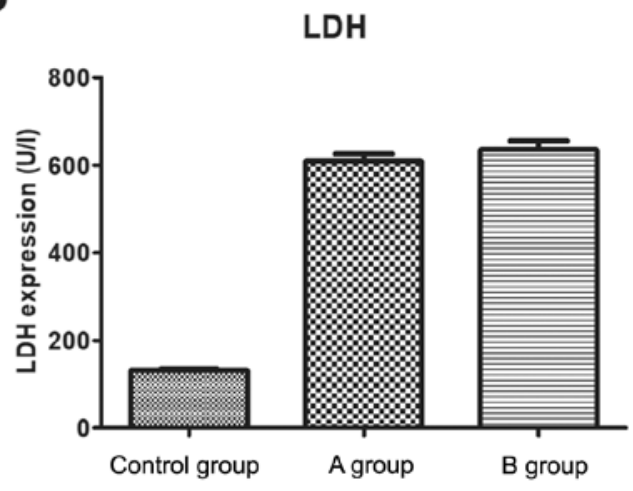

D

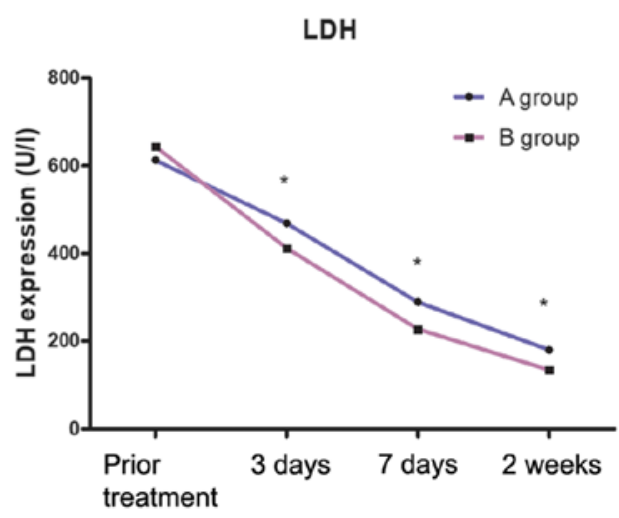

Figure 1. Comparison of the CK-MB and LDH levels of myocardial enzymes at different time points before and after treatment. (A and B) Concentrations of CK-MB and LDH in the RG were significantly higher than those in the $\mathrm{CG}(\mathrm{P}<0.01)$ before treatment, and there was no significant difference between groups $\mathrm{A}$ and $\mathrm{B}(\mathrm{P}>0.05)$. (C and $\mathrm{D})$ The levels of CK-MB and LDH in group A and group B at different time points decreased 3 days, 7 days and 2 weeks after treatment, respectively, but the decrease level in group $\mathrm{B}$ was better than that in group $\mathrm{A}\left({ }^{*} \mathrm{P}<0.05\right)$. CK-MB, creatine kinase isoenzyme; $\mathrm{LDH}$, lactate dehydrogenase; $\mathrm{LDH}$, lactate dehydrogenase; $\mathrm{RG}$, research group; $\mathrm{CG}$, control group.

family history of heart disease, or smoking in ACOP patients $(\mathrm{P}>0.05)$, but correlated with co-exposure time and typing $(\mathrm{P}<0.05)($ Table V).

\section{Discussion}

$\mathrm{CO}$ is a common fatal poison that easily damage the transportation and utilization of oxygen (5). Mild ACOP symptoms include dizziness, headache, and limb weakness. Long-term exposure to $\mathrm{CO}$ can lead to central nervous system and cardiovascular system injury and even death $(10,11)$. ACOP has become one of the most common types of poisoning causing death in the world (12). As the target organ of
ACOP, heart failure is the main clinical manifestation after poisoning (13). At present, the determination of myocardial enzyme is the main clinical indicator of myocardial cell injury (14). However, due to abnormal expression of myocardial enzyme in various cardiac diseases and low specificity, it is of great significance to find markers for diagnosing ACOP cardiac injury. MircoRNA is a kind of small molecule non-coding RNA; previous studies have shown that miR-30a participates in the occurrence and development of cardiovascular diseases, and plays an important role in myocardial function, myocardial cell necrosis and apoptosis by regulating its target genes (15), proving that miR-30a is abnormally expressed in blood of patients with myocardial 

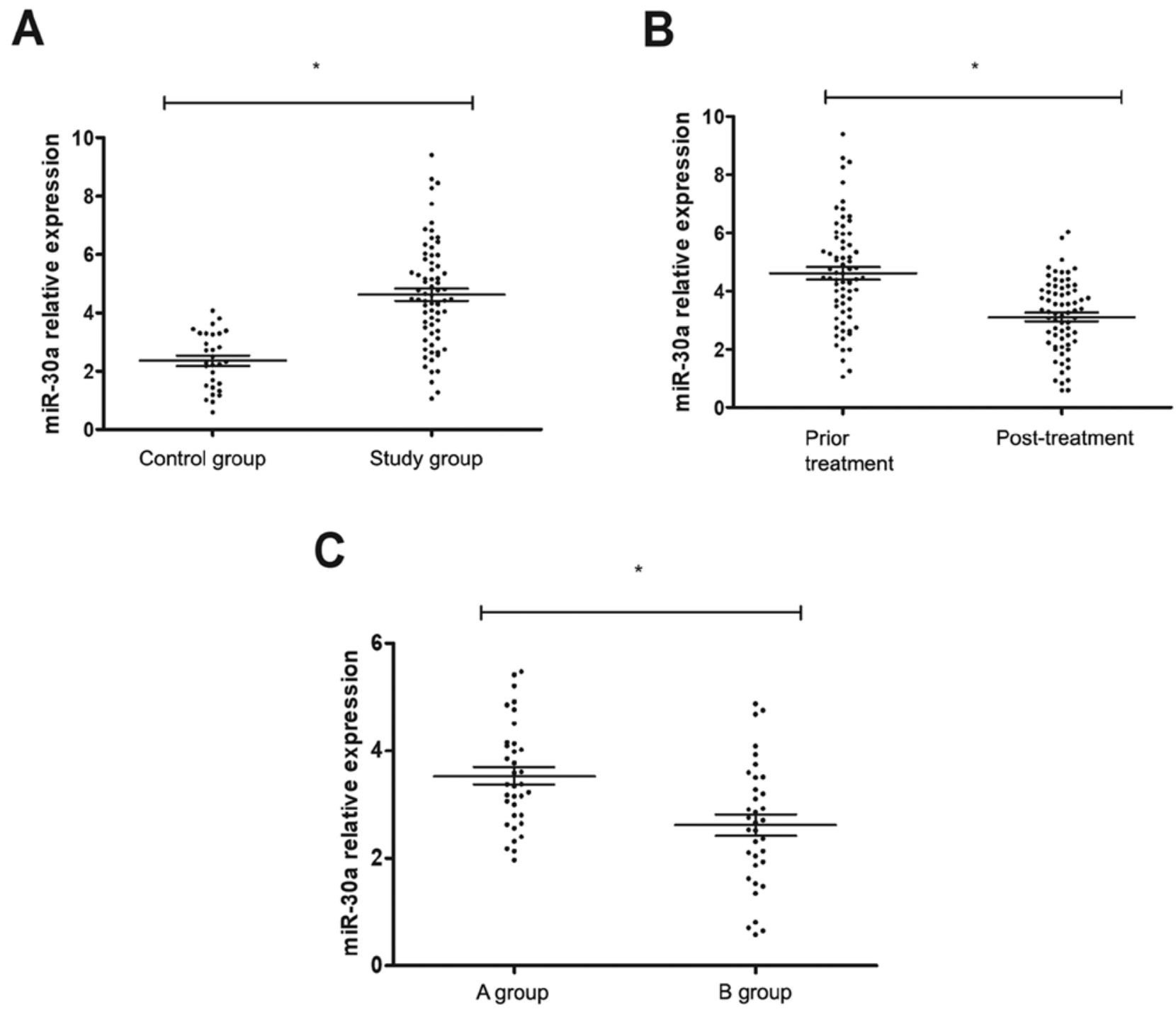

Figure 2. Comparison of the miR-30a expression level before and after treatment. (A) The relative expression level of miR-30a in the RG before treatment $(4.51 \pm 1.63)$ was significantly higher than that in the CG (2.15 \pm 1.02$)$; (B) The relative expression level of miR-30a (3.16 \pm 1.24$)$ in the RG decreased significantly 7 days after treatment compared with that before treatment. (C) The relative expression level of miR-30a in the two groups after treatment with different treatment methods, show that the relative expression level of miR-30a in group A was $3.35 \pm 1.03$, that in group B was $2.86 \pm 0.95$, and that in group A was significantly lower than that in group B ("P<0.05). RG, research group; CG, control group.

infarction (16). At present, the treatment of ACOP includes giving high concentration and high flow of oxygen, ventilation support and detection of abnormal heart rate (17). Hyperbaric oxygen is an effective treatment method of ACOP, which can quickly correct anoxia. Although hyperbaric oxygen treatment is related to the good consciousness level of $\mathrm{CO}$ poisoning patients, its impact on their mortality or morbidity rate is still unknown, and is not directly tied to their reduction of mortality rate (18). However, in ACOP multiple organ failure of adults and children, there have been cases showing success of extracorporeal membrane oxygenation (ECMO) treatment (19). ECMO is an extracorporeal circulation technology and has been clinically developed as a necessary means to treat respiratory and circulatory failure (20). Since there are few reports comparing the efficacy of the two therapeutic methods on ACOP, this study compared the efficacy of hyperbaric oxygen therapy and ECMO on ACOP patients, respectively, and detects the expression of miR-30a in cardiac injury of ACOP, proving the application value of the two therapeutic methods, which has important reference value for clinical ACOP treatment.

In this study, 70 patients with confirmed moderate or severe ACOP were evaluated, the effects of hyperbaric oxygen therapy and ECMO therapy were compared, and the myocardial enzyme CK-MB, LDH concentration and miR-30a expression in peripheral blood in ACOP-induced cardiac injury were detected. The results showed that ECMO was superior to hyperbaric oxygen in terms of treatment efficiency and complication rate, and the reason was that it could remove $\mathrm{CO}$ in patients and improve hypoxia state of their tissues and organs more effectively, which was consistent with the research results of Choi et al (21). Furthermore, CK-MB and LDH concentrations in ACOP patients increased significantly, but decreased significantly three days, seven days and two weeks after treatment, respectively. However, the degree of the decrease in the ECMO group was better than that in hyperbaric oxygen group. RT-PCR was used to detect the miR-30a expression level in the blood of the study subjects. It was found that the miR-30a 
Table V. Correlation analysis of the miR-30a expression level and clinicopathological features of patients in the RG.

\begin{tabular}{|c|c|c|c|c|}
\hline Parameters & $\mathrm{n}$ & miR-30a level & $\mathrm{t}$ value & P-value \\
\hline \multicolumn{5}{|l|}{ Age (years) } \\
\hline$\geq 41.5$ & 41 & $4.23 \pm 1.17$ & 0.445 & 0.658 \\
\hline$<41.5$ & 29 & $4.36 \pm 1.25$ & & \\
\hline Sex & & & 0.349 & 0.729 \\
\hline Male & 33 & $4.06 \pm 1.12$ & & \\
\hline Female & 37 & $3.97 \pm 1.04$ & & \\
\hline Exposure time (h) & & & 2.123 & 0.037 \\
\hline$\geq 6.5$ & 28 & $4.56 \pm 1.41$ & & \\
\hline$<6.5$ & 42 & $3.93 \pm 1.07$ & & \\
\hline Typing & & & 2.180 & 0.033 \\
\hline Moderate & 44 & $4.17 \pm 1.16$ & & \\
\hline Severe & 26 & $4.82 \pm 1.28$ & & \\
\hline Family history of heart disease & & & 0.815 & 0.418 \\
\hline Yes & 11 & $4.35 \pm 1.37$ & & \\
\hline No & 59 & $4.07 \pm 0.98$ & & \\
\hline Smoking & & & 0.545 & 0.588 \\
\hline Yes & 26 & $4.26 \pm 1.15$ & & \\
\hline No & 44 & $4.11 \pm 1.09$ & & \\
\hline
\end{tabular}

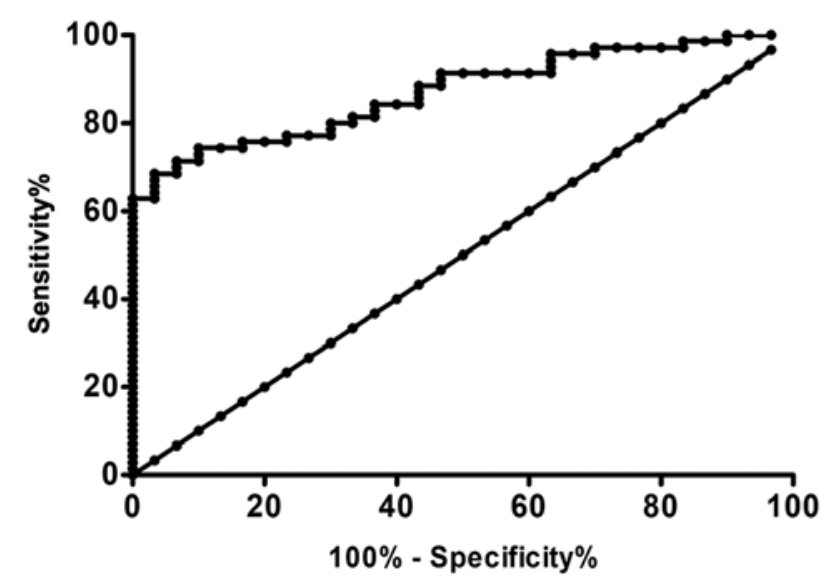

Figure 3. Early diagnostic value of miR-30a for myocardial injury of ACOP (AUC=0.870). ACOP, acute carbon monoxide poisoning; AUC, area under the curve.

expression level in the blood of ACOP patients was significantly different from that of the normal control group, and that of the ACOP patients were significantly higher than normal people. Seven days after treatment, the expression of miR-30a in group A and group B decreased significantly compared with that before, but the decrease in group B was more obvious, which showed that ECMO was better than hyperbaric oxygen therapy in treating cardiac injury. ROC curve analysis showed that the cut-off value was 3.807 , miR-30a had a sensitivity of $68.57 \%$ and specificity of $96.67 \%$ in diagnosing cardiac injury of ACOP, indicating that miR-30a had certain accuracy.
Through further analysis of the correlation between miR-30a and clinical pathology of ACOP patients, we found that the miR-30a expression level was not linked to age, sex, family history of heart disease, or smoking, but linked to time of exposure to $\mathrm{CO}$ and ACOP typing, suggesting that miR-30a played an important part in the proliferation and apoptosis of myocardial cells under anoxic conditions, and myocardial cell damage and recovery could be judged by detecting the miR-30a expression level in peripheral blood.

Collectively, hyperbaric oxygen therapy and ECMO therapy have obvious efficacy on ACOP patients, but the latter is better than the former, and the expression level of miR-30a in blood of ACOP patients increased significantly, which is positively correlated with myocardial injury, and it decreased after treatment. It is believed that miR-30a can provide a reference index for early diagnosis and prediction of disease progression and prognosis in cardiac injury of ACOP.

\section{Acknowledgements}

Not applicable.

\section{Funding}

No funding was received.

\section{Availability of data and materials}

The datasets used and/or analyzed during the current study are available from the corresponding author on reasonable request. 


\section{Authors' contributions}

SG wrote the manuscript. $\mathrm{XH}$ and $\mathrm{HXu}$ conceived and designed the study. JY and DH contributed to observation indexes analysis. SG and HXin interpreted the data and drafted the manuscript. XG and RZ performed PCR and ELISA. All authors read and approved the final manuscript.

\section{Ethics approval and consent to participate}

The study was approved by the Ethics Committee of The Affiliated Hospital of Qingdao University (Qingdao, China). Patients who participated in this study had complete clinical data. Signed informed consents were obtained from the patients and/or guardians.

\section{Patient consent for publication}

Not applicable.

\section{Conflict of interest}

The authors declare that they have no competing interests.

\section{References}

1. Xiang W, Xue H, Wang B, Li Y, Zhang J, Jiang C, Liang F Pang $\mathrm{J}$ and $\mathrm{Yu} \mathrm{L}$ : Combined application of dexamethasone and hyperbaric oxygen therapy yields better efficacy for patients with delayed encephalopathy after acute carbon monoxide poisoning. Drug Des Devel Ther 11: 513-519, 2017.

2. Rose JJ, Wang L, Xu Q, McTiernan CF, Shiva S, Tejero J and Gladwin MT: Carbon monoxide poisoning: Pathogenesis, management, and future directions of therapy. Am J Respir Crit Care Med 195: 596-606, 2017

3. Oh S and Choi SC: Acute carbon monoxide poisoning and delayed neurological sequelae: A potential neuroprotection bundle therapy. Neural Regen Res 10: 36-38, 2015.

4. Lee FY, Chen WK, Lin CL and Kao CH: Carbon monoxide poisoning and subsequent cardiovascular disease risk: A nationwide population-based cohort study. Medicine (Baltimore) 94: e624, 2015.

5. Clardy PF, Manaker S and Perry H: Carbon monoxide poisoning. UpToDate 2018. https://www.uptodate.com/contents/carbonmonoxide-poisoning.

6. Huang CC, Ho CH, Chen YC, Lin HJ, Hsu CC, Wang JJ, Su SB and Guo HR: Hyperbaric oxygen therapy is associated with lower short- and long-term mortality in patients with carbon monoxide poisoning. Chest 152: 943-953, 2017.

7. Eichhorn L, Thudium $M$ and Jüttner B: The diagnosis and treatment of carbon monoxide poisoning. Dtsch Arztebl Int 115: 863-870, 2018

8. Simonsen C, Magnusdottir SO, Andreasen JJ, Rohde MC and Kjærgaard B: ECMO improves survival following cardiogenic shock due to carbon monoxide poisoning - an experimental porcine model. Scand J Trauma Resusc Emerg Med 26: 103, 2018 .
9. Yang Y, Li Y, Chen X, Cheng X, Liao Y and Yu X: Exosomal transfer of miR-30a between cardiomyocytes regulates autophagy after hypoxia. J Mol Med (Berl) 94: 711-724, 2016.

10. Lin MS, Lin CC, Yang CC, Weng SC, Wang SM, Chen CY, Huang $\mathrm{N}$ and Chou YH: Myocardial injury was associated with neurological sequelae of acute carbon monoxide poisoning in Taiwan. J Chin Med Assoc 81: 682-690, 2018.

11. Lin CH, Su WH, Chen YC, Feng PH, Shen WC, Ong JR, Wu MY and Wong CS: Treatment with normobaric or hyperbaric oxygen and its effect on neuropsychometric dysfunction after carbon monoxide poisoning: A systematic review and meta-analysis of randomized controlled trials. Medicine (Baltimore) 97: e12456, 2018.

12. Gozubuyuk AA, Dag H, Kaçar A, Karakurt Y and Arica V: Epidemiology, pathophysiology, clinical evaluation, and treatment of carbon monoxide poisoning in child, infant, and fetus. North Clin Istanb 4: 100-107, 2017.

13. Garg J, Krishnamoorthy P, Palaniswamy C, Khera S, Ahmad H, Jain D, Aronow WS and Frishman WH: Cardiovascular abnormalities in carbon monoxide poisoning. Am J Ther 25: e339-e348, 2018.

14. Maghamiour N and Safaie N: High creatine kinase (CK)-MB and lactate dehydrogenase in the absence of myocardial injury or infarction: A case report. J Cardiovasc Thorac Res 6: 69-70, 2014.

15. Huang J, Huang C, Luo Y, Liu S and Chen X: Role of miR-30a in cardiomyocyte autophagy induced by angiotensin II. J Renin Angiotensin Aldosterone Syst 16: 1-5, 2015.

16. Wang Y, Huang Y, Zhang M, Zhang X, Tang X and Kang Y: Bioinformatic analysis of the possible regulative network of miR-30a/e in cardiomyocytes 2 days post myocardial infarction. Acta Cardiol Sin 34: 175-188, 2018.

17. Casillas S, Galindo A, Camarillo-Reyes LA, Varon J and Surani SR: Effectiveness of hyperbaric oxygenation versus normobaric oxygenation therapy in carbon monoxide poisoning: A systematic review. Cureus 11: e5916, 2019.

18. Nakajima M, Aso S, Matsui H, Fushimi $\mathrm{K}$ and Yasunaga $\mathrm{H}$ : Hyperbaric oxygen therapy and mortality from carbon monoxide poisoning: A nationwide observational study. Am J Emerg Med S0735-6757(19)30087-7, 2019.

19. Rabie AA, Asiri A, Alsherbiny M, Alqassem W, Rajab M, Mohamed S, I Alenazi WH and Ariplackal L: Management of carbon monoxide poisoning-induced cardiac failure and multiorgan dysfunction with combined respiratory and circulatory extracorporeal membrane oxygenation. Saudi Crit Care J 3: $12,2019$.

20. Fan E, Gattinoni L, Combes A, Schmidt M, Peek G, Brodie D, Muller T, Morelli A, Ranieri VM, Pesenti A, et al: Venovenous extracorporeal membrane oxygenation for acute respiratory failure: A clinical review from an international group of experts. Intensive Care Med 42: 712-724, 2016.

21. Choi JH, Kim SW, Kim YU, Kim SY, Kim KS, Joo SJ and Lee JS: Application of veno-arterial-venous extracorporeal membrane oxygenation in differential hypoxia. Multidiscip Respir Med 9: 55,2014 .

This work is licensed under a Creative Commons Attribution-NonCommercial-NoDerivatives 4.0 International (CC BY-NC-ND 4.0) License. 